\title{
Addressing adolescent alcohol use in South Africa
}

\author{
N K Morojele, ${ }^{1,2} \mathrm{PhD} ;$ L Ramsoomar, ${ }^{2} \mathrm{PhD}$ \\ ${ }^{1}$ Alcohol, Tobacco and Other Drug Research Unit, South African Medical Research Council, Pretoria; \\ and School of Public Health and Family Medicine, Faculty of Health Sciences, University of Cape Town, South Africa \\ ${ }^{2}$ School of Public Health, Faculty of Health Sciences, University of the Witwatersrand, Johannesburg, South Africa
}

Corresponding author: N K Morojele (neo.morojele@mrc.ac.za)

Excessive alcohol consumption constitutes a significant public health problem for South Africans. Alcohol use by South African (SA) adolescents is characterised mainly by binge/heavy episodic drinking. Levels of binge drinking have been high, but relatively stable, among males since 2002, while there has been a significant increase in binge drinking by females since then. Binge drinking is a major risk factor for a range of alcohol-related harms in SA, including traffic-related accidents and deaths, interpersonal violence, fetal alcohol spectrum disorder (FASD), crime, sexual risk behaviour, HIV, tuberculosis and the resultant burden of all of these on the economy. Clinicians may play a key role in addressing adolescent alcohol use and alcohol-related harm. Such a role may involve screening, brief interventions and referrals to treatment. There are several assessment, screening and diagnostic tools to detect alcohol use and misuse, specifically among adolescents. Furthermore, various pharmacological and psychological approaches are available to treat adolescent alcohol problems. Special issues to consider when dealing with alcohol use problems among adolescents in SA include recognising the risk factors, and acknowledging and addressing the harms associated with alcohol use (including sexual risk behaviour and FASD) and the possible existence of comorbid mental health problems.

S Afr Med J 2016;106(6):551-553. DOI:10.7196/SAMJ.2016.v106i6.10944

\section{Definitions}

- Adolescents are defined as young people aged 10 - 19 years. ${ }^{[1]}$

- Alcohol use disorder (AUD) constitutes a combination of alcohol use and alcohol dependence in a single disorder, according to the Diagnostic and Statistical Manual of Mental Disorders, 5th edition (DSM-5). ${ }^{[2]}$ AUD is diagnosed when someone presents with the harmful use of alcohol, social and interpersonal problems related to alcohol use, and neglect of major roles owing to use. Furthermore, a diagnosis is made when someone presents with at least two of 11 criteria in the past 12 months. ${ }^{[2]}$ The greater the number of criteria met, the greater the severity of the disorder. ${ }^{[2]}$ The recent transition from the DSM-IV to DSM-5 has removed the previous distinction between alcohol abuse and alcohol dependence, as problems with alcohol are assumed to exist on a continuum. AUDs can be mild ( 2 - 3 symptoms), moderate (4 - 5 symptoms), or severe ( $\geq 6$ symptoms).

- Binge/heavy episodic drinking refers to the consumption, on one occasion, of $\geq 5$ drinks for males and $\geq 4$ for females.

\section{Introduction and epidemiology}

Excessive alcohol consumption constitutes a significant public health problem for South Africans. The current drinking situation in South Africa (SA) is characterised by abstinence from alcohol use juxtaposed with heavy episodic drinking. From a global perspective, almost half of the SA population (42.0\% of people $>15$ years of age) abstain from alcohol during their lifetime, $17.3 \%$ are former drinkers, and $59.4 \%$ have abstained from drinking during the past 12 months. Females abstain more than males in all categories of abstinence. ${ }^{[1]}$

The patterns of drinking that are common among South Africans are of major concern for public health. Apart from achieving global status as one of the most risky drinking countries, ${ }^{[3]}$ a recent national review indicated the high levels of binge/heavy episodic drinking. ${ }^{[4]}$ The study specifically found that the prevalence of hazardous or harmful drinking among current drinkers was $31.5 \%$ (39.4\% among males and $16.6 \%$ among females). ${ }^{[4]}$ There are very clear variations among South Africans with regard to drinking, most notably by gender, race and province.

The most recent World Health Organization (WHO) Global Status Report on Alcohol and Health indicated a prevalence of $5.6 \%$ of AUDs among SA adults and adolescents (10.0\% among males and $1.5 \%$ among females), ${ }^{[1]}$ and a prevalence of $2.4 \%$ for alcohol dependence ( $4.2 \%$ for males and $0.7 \%$ for females). ${ }^{[1]}$

\section{Drinking among SA} adolescents

Adolescence represents a critical developmental period during which young people often experiment with and initiate alcohol use. It is also a period when several physiological changes occur in the brain, physical changes occur in the body (e.g. onset of puberty) and young people experience other sociopsychological transitions. Lifetime alcohol use among adolescents in SA can be characterised as stable but high during 2002 - 2011, with age of initiation $<13$ years having been stable at $12 \%$ over the same period (Table 1) ${ }^{[4]}$ Drinking among SA adolescents is characterised by binge/heavy episodic drinking. ${ }^{[1,5]}$ According to the national Youth Risk Behaviour Survey (YRBS), binge drinking

Table 1. SA adolescents' alcohol consumption by gender: findings from the YRBS ${ }^{[5-7]}$

\begin{tabular}{|c|c|c|c|c|c|c|}
\hline \multirow[b]{2}{*}{ Alcohol consumption } & \multicolumn{2}{|c|}{2002} & \multicolumn{2}{|c|}{2008} & \multicolumn{2}{|c|}{2011} \\
\hline & Male & Female & Male & Female & Male & Female \\
\hline Ever used alcohol, \% & 56.1 & 43.5 & 54.4 & 45.1 & 53.8 & 44.9 \\
\hline Current alcohol use (past 30 days), $\%$ & 38.5 & 26.4 & 40.5 & 29.5 & 36.6 & 28.2 \\
\hline Binge drinking during past month, $\%$ & 29.3 & 17.9 & 33.5 & 23.7 & 30.3 & 20.1 \\
\hline Age of initiation $<13$ years, $\%$ & 15.8 & 9.0 & 15.3 & 8.6 & 16.3 & 8.7 \\
\hline
\end{tabular}


increased between 2002 and 2008 and stabilised in 2011 among males. For females there was a significant increase between 2002 and 2008, which was not sustained in 2011

\section{Consequences of adoles- cent drinking in SA}

Binge drinking has important implications for long-term progression to problem drinking and acute alcohol-related harms, highlighted in both the global and the national literature. ${ }^{[1,4,7]}$ Binge drinking is a major risk factor for a range of alcohol-related harms in SA, including traffic-related accidents and deaths, interpersonal violence, fetal alcohol syndrome, crime, sexual risk, tuberculosis, pneumonia and the resultant burden of all these harms on the economy. ${ }^{[8]}$ The most recent findings of the South African Community Epidemiological Network on Drug Use (SACENDU) revealed that, with the exception of one site, between $11 \%$ and $19 \%$ of patients being treated were $<20$ years of age. ${ }^{[9]}$ In light of SA's status as one of the countries with the most risky drinking patterns, it is essential to address binge and problem drinking early in life to prevent both its direct effects on individual health and wellbeing and the associated harms. The WHO Global Status Report on Alcohol and Health highlights the important role of health professionals in 'monitoring alcohol consumption in their patients and providing brief interventions, counselling and pharmacotherapy, as appropriate, in all cases of identified hazardous drinking or alcohol use disorder. ${ }^{[1]}$ Given the ubiquity of alcohol consumption in SA, it is important that clinicians regard alcohol and other drug use problems as central to patient care.

\section{Assessment, screening and diagnosis}

Since adolescents are often not readily inclined to seek treatment for problems related to their alcohol use, routine screening is recommended. ${ }^{[10]}$ Screening, brief interventions and referral to treatment is advised, particularly to allow for early intervention, and to prevent alcohol-related harms and the progression to more serious AUDs.

There are several assessment, screening and diagnostic tools to detect alcohol use and misuse. However, not all of these are recommended for adolescent populations. Among the tools applicable to adolescent populations in primary care are: the Alcohol Use Disorders Identification Test (AUDIT), its shorter adaption (AUDIT-C) (Box 1), the CRAFFT (Car, Relax, Alone, Forget, Family or Friends, and Trouble) instrument
(Box 2), and the Problem Oriented Screening Instrument for Teenagers (POSIT). ${ }^{[1]}$

The AUDIT has been validated for identifying adolescents at risk for alcohol misuse. However, a practical challenge of administering the AUDIT, particularly in busy clinical settings, is its length (10 items). Therefore, a shorter version of the AUDIT (AUDIT-C) is the preferred instrument, comprising only the first three items, which assess different aspects of alcohol consumption (Box 1)

The AUDIT-C is scored on a scale of 0 12 , with 0 indicating the absence of alcohol problems. Gender-specific scoring has been applied, with a score of $\geq 4$ for men and $\geq 3$ for women considered to be optimal for identifying hazardous drinking or active AUDs.

A recent review of the CRAFFT concluded that it had adequate psychometric properties

\section{Box 1. Items of the AUDIT-C}

How often do you have a drink containing alcohol?
Never
Monthly or less
2 - 4 times a month
2 - 3 times a week
$\geq 4$ times a week

How many standard drinks containing alcohol do you have on a typical day when drinking?

1 or 2

3 or 4

5 or 6

$7-9$

$\geq 10$

How often do you have $\geq 6$ drinks on one occasion?

Never

Less than monthly

Monthly

Weekly

Daily or almost daily

AUDIT-C $=$ shorter version of the AUDIT.

\section{Box 2. Items of the CRAFFT}

- Have you ever ridden in a Car driven by someone else (or yourself) who was 'high' or had been using alcohol and drugs?

- Do you drink to Relax?

- Do you ever drink while you are Alone?

- Do you ever Forget things you did while using alcohol or drugs?

- Do any of your closest Family or Friends ever tell you that you should cut down on your drinking?

- Have you ever gotten into Trouble while you were using alcohol or drugs? for the detection of AUDs among adolescents $^{[12]}$ and is widely used to detect alcohol misuse in this age group. CRAFFT is an acronym that consists of six operative words as indicated in Box 2. A score of $\geq 2$ on the CRAFFT is indicative of the presence of an alcohol- or drug-related problem.

The POSIT consists of 139 items relating to 10 different problem areas, including alcohol and other drug use, mental health problems and other psychosocial problems. It is most suitable for more comprehensive screening in settings where time constraints are not a major concern.

\section{Treatment and management}

\section{Brief interventions}

Brief interventions are generally of short duration and can be effective for use as part of universal and/or selective and/or indicated prevention approaches. ${ }^{[13]}$ These interventions can be delivered by different categories of healthcare providers, including physicians, nurses, social workers and psychologists. According to a recent metaanalysis, brief interventions are effective (with modest effects) for reducing alcohol consumption and alcohol-related harm among adolescents. ${ }^{[13]}$ Such improvements were sustained for as long as 1 year, although the studies in the review did not have long follow-up periods for adolescents. The review included interventions of up to 5 hours' contact time, and a maximum of 4 weeks between the first and last intervention session (with booster sessions in some cases). Overall, brief interventions were found to be effective regardless of intervention modality (e.g. cognitive behavioural therapy (CBT) only, or motivational enhancement therapy (MET) only, or MET/CBT, or feedback/ information); delivery site (primary care/ health centre, or school/university, or emergency room or self-administered); or format (alone, individual, or group). However, interventions that included decisional balance and/or goal-setting exercises were particularly effective, and brief interventions had stronger effects on alcohol-related problems among younger adolescents than older ones Finally, the review indicated that adolescents who were identified as high risk may benefit more than those not so identified (i.e. non-drinkers or low-risk drinkers).

\section{Specialised treatment}

Where referral is needed, adolescent patients may receive a range of treatments, comprising psychosocial treatments alone or in combination with pharmacotherapy. The most com- 
mon types of psychosocial treatments for adolescents have been identified as: (i) family-based therapy; (ii) individual and group therapy - these mainly include CBT, brief interventions and motivational approaches, and contingency management reinforcement approaches; (iii) 12-step programmes; and (iv) therapeutic communities. ${ }^{[14]}$

Different types of pharmacological therapies may be used for treating and managing AUDs. According to Clark, ${ }^{[15]}$ these are most effective in conjunction with psychotherapy and are used for addressing withdrawal, reducing craving, minimising alcohol's reinforcing properties, and/or addressing other mental health conditions. Alcohol withdrawal, which is extremely rare among adolescents, is generally treated using benzodiazepines, as in adults. These drugs are assumed to be appropriate for adolescents, but such use is not supported by empirical evidence. ${ }^{[15]}$ Benzodiazepines are best used under medical supervision and with caution in pregnant women, given their teratogenic effects. Disulfiram (Antabuse) is used to increase physical aversion to alcohol, as it gives rise to negative symptoms (flushing, nausea, vomiting) when used with alcohol. ${ }^{[15]}$ It is rarely used for adolescents, and requires close supervision and high levels of adherence to abstinence, particularly given the effects (arrhythmias, hypotension ${ }^{[15]}$ ) when alcohol is consumed in large quantities. ${ }^{[15]}$ The most common medications for reducing craving are acamprosate and naltrexone. ${ }^{[15]}$ Evidence for the effectiveness of these drugs in adolescents is limited. ${ }^{[15]}$ Finally, certain pharmacotherapies are recommended for treating common comorbid conditions, such as attention-deficit hyperactivity disorder, major depressive disorder, conduct disorder and posttraumatic stress disorder. Treatment of these disorders may improve the outcomes of AUD treatment, and may include antidepressant and stimulant medications. ${ }^{[15]}$ However, the limited evidence to support the use of these medications for adolescents and their abuse potential should be taken into account when they are being

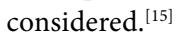

In spite of the availability of a range of treatment approaches for addressing alcohol use and other disorders among adolescents, many of the existing services have several shortcomings, and access to treatment is often difficult in SA. ${ }^{[8]}$ SA has very few adolescent-focused or adolescent-exclusive treatment centres, and many existing services do not employ evidence-based approaches. Based on the limitations identified ${ }^{[8]}$ it is recommended that several factors, such as whether or not the service is adolescent focused, whether or not the programme is evidence based, and the extent of family involvement, should be considered when referring adolescents for treatment.

\section{Special considerations Screening}

Factors that may influence decisions about whom to screen may depend on whether the interventions involve a universal, selected or indicated approach. Moreover, in a high-risk community, where the prevalence of AUDs is relatively high, routine screening may be particularly important. One may also consider the presence of other individual risk factors, including a family history of alcohol problems and other mental health problems, e.g. risk factors, when deciding whom to screen and target for further assessment and treatment.

\section{Treatment goal(s)}

Total abstinence is generally accepted as the most appropriate treatment goal for adolescents with an AUD. The findings of a number of studies showing cardioprotective effects of alcohol may tempt the clinician to suggest moderate drinking as an ideal treatment goal. However, these studies have not typically involved adolescents, and the effects have been observed to diminish in cases of heavy episodic drinking. ${ }^{[1]}$ Consequently, for all adolescents, the message that a little drinking may be good for them is best avoided, while the conclusions with regard to adults are more open to debate.

\section{Comorbidity}

Adolescents who engage in heavy drinking or are identified as having an AUD are typically at increased risk of other mental health, social or behavioural problems. For example, alcohol increases the risk of engagement in sexual risk behaviour, leading to sexually transmitted infections (STIs), including HIV. It is important to highlight these risks when engaging with adolescents, and to refer them for further assessment to screen for possible STIs, including HIV, where indicated.

\section{Adolescent girls}

Adolescent girls who drink alcohol are also at increased risk of unprotected sex, unplanned pregnancy and sexual assault. Alcohol consumption during pregnancy increases the risk of development of fetal alcohol spectrum disorders (FASDs) in the unborn child. Clinicians are advised to inform sexually active adolescents about the risk of FASDs, and to recommend total abstinence from alcohol among those who are not using contraceptives, given the current uncertainty about the exact threshold (if any) at which drinking becomes harmful.

\section{Summary}

Excessive alcohol consumption constitutes a significant public health problem among adolescents in SA. General practitioners are well placed to screen, treat and/or refer them for alcohol-related treatment. Several screening tools are available to guide practitioners on how best to manage their patients, while psychosocial and pharmacological approaches are also available (the latter under medical supervision) for the few instances in which they may be required. Notwithstanding time and other constraints in their busy practices, routine screening to detect alcohol use among adolescents and early intervention by physicians are highly recommended.

\section{References}

1. World Health Organization. Global Status Report on Alcohol and Health. Geneva: WHO, 2014:20. http://apps. who.int/iris/handle/10665/112736 (accessed 22 June 2014).

2. American Psychiatric Association. Diagnostic and Statistical Manual of Mental Disorders. 5th ed. American Psychiatric Association. Diagnostic and Sta

3. Peltzer K, Davids A, Njuho P. Alcohol use and problem drinking in South Africa: Findings from a national population-based survey. Afr J Psychiatry (Johannesburg) 2011;14(1):30-37.

4. Ramsoomar L, Morojele NK. Trends in alcohol prevalence, age of initiation and association with alcoholrelated harm among South African youth: Implications for policy. S Afr Med J 2012;10:609-612.

5. Reddy SP, James S, Sewpaul R, et al. Umthente Uhlaba Usamila - the 3rd South African National Youth Risk Behaviour Survey 2011. Cape Town: South African Medical Research Council, 2013

6. Reddy SP, James S, Sewpaul R, et al. Umthente Uhlaba Usamila - the 2nd South African Youth Risk Behaviour Survey 2008. Cape Town: South African Medical Research Council, 2010.

Reddy SP, James S, Sewpaul R, et al. Umthente Uhlaba Usamila - the 1st South African Youth Risk Behaviour Survey 2002. Cape Town: South African Medical Research Council, 2003.

8. Morojele N, Rich E, Flisher A, Myers B. Youth and substances. In: Ellis G, Stein D, Meintijes E, Thomas K, eds. Substance Use and Abuse in South Africa: Insights from Brain and Behavioural Sciences. Cape Kown: University of Cape Town, 2012:231-256.

9. Dada S, Harker Burnhams N, Erasmus J, et al. South African Community Network on Drug Use (SACENDU): Monitoring Alcohol, Tobacco and Other Drug Abuse Trends in South Africa (July (SACENDU): Monitoring Alcohol, Tobacco and Other Drug Abuse Trends in South Africa (July -
December 2014). Cape Town: South African Medical Research Council, 2015. http://www.mrc.ac.za/ adarg/sacendu/SacenduUpdateJune2015.pdf (accessed 11 September 2015).

10. Mitchell SG, Gryczynski J, O'Grady KE, et al. SBIRT for adolescent drug and alcohol use: Curren status and future directions. J Subst Abuse Treat 2013;44(5):463-472. DOI:10.1016/j.jsat.2012.11.005

11. Rumpf HJ, Wohlert T, Freyer-Adam J, Grothues J, Bischof G. Screening questionnaires for problem drinking in adolescents: Performance of AUDIT, AUDIT-C, CRAFFT and POSIT. Eur Addict Res 2013;19(3):121-127. DOI:10.1159/000342331

12. Mitchell SG, Kelly SM, Gryczynski J, et al. The CRAFFT cut-points and DSM-5 criteria for alcoho and other drugs: A re-evaluation and re-examination. Subst Abus 2014;35(4):376-380. DOI:10.1080 /08897077.2014.936992

13. Tanner-Smith EE, Lipsey MW. Brief alcohol interventions for adolescents and young adults: A systematic review and meta-analysis. J Subst Abuse Treat 2015;51:1-18. DOI:10.1016/j.jsat.2014.09.001

14. Winters KC, Botzet AM, Fahnhorst T. Advances in adolescent substance abuse treatment. Curr Psychiatry Rep 2011;13(5):416-421. DOI:10.1007/s11920-011-0214-2

15. Clark DB. Pharmacotherapy for adolescent alcohol use disorder. CNS Drugs 2012;26(7):559-569. DOI:10.2165/11634330-000000000-00000 